\title{
ORACIONES CAUSATIVAS EN EL INGLÉS CRIOLLO DE COSTA RICA
}

\author{
Mario Portilla
}

\begin{abstract}
RESUMEN
El presente artículo describe las diferentes estrategias que se utilizan en el inglés criollo de Costa Rica para expresar las predicaciones causativas. Por un lado, las construcciones causativas analíticas presentan la aplicación de varias reglas sintácticas que permiten la inserción de completizadores, movimientos de topicalización y movimientos de foco o contraste. Por otro lado, en las construcciones causativas léxicas, se describen tres operaciones morfológicas que sufren los verbos no causativos cuando se convierten en verbos causativos.
\end{abstract}

\begin{abstract}
This paper describes two different strategies that are used in Costa Rican Creole English to express causative predications. On one hand, analytic causatives involve several syntactic operations that allow insertion of complementizers, topicalization, and focus or contrast movements. On the other hand, in the case of lexical causatives, three different morphological operations that occur when noncausative verbs are converted into causatives are discussed.
\end{abstract}

\section{Construcciones causativas}

Una construcción causativa consiste en una expresión lingüística que contiene la predicación de una causa y la predicación de un argumento que expresa un efecto (Payne 1997: 176). En términos lógicos, la relación entre estas predicaciones se puede simbolizar de la siguiente manera: CAUSAR $(\mathrm{x}, \mathrm{P})=\mathrm{x}$ causa $\mathrm{P}^{1}$.

En español, un ejemplo que ilustra esta relación es el siguiente:

HACER (Juan, LLORAR (María)) = Juan hizo que María llorara.

En estas construcciones se pueden distinguir, por lo tanto, un predicado de la causa, el cual contiene la noción de causalidad, y un predicado del efecto, el cual expresa el resultado de la situación causativa. En el ejemplo anterior, el predicado de la causa está expresado por la fórmula: HACER (Juan), y el predicado del efecto por: LLORAR (María).

El agente del predicado de la causa y también de la situación causativa se suele denominar el agente de la causa (causer). El agente del evento causado se puede llamar el 
predicador del efecto (causee). En el ejemplo citado, JUAN es el agente de la causa y MARíA, el predicador del efecto.

Como es evidente, la expresión del predicado de la causa significa la adición de un nuevo participante a la escena que se manifiesta en el predicado del efecto, es decir, la introducción del agente de la causa.

En las diferentes lenguas, existen tres tipos de estrategias para expresar predicaciones causativas: una léxica, una morfológica y una analítica. En el inglés criollo de Costa Rica, solamente se han podido identificar construcciones causativas analíticas y construcciones causativas léxicas. Esto se debe, sin duda, a la ausencia prácticamente total de morfología flexiva en este idioma, un rasgo característico de la mayoría de las lenguas criollas.

El presente trabajo tiene como objetivo describir las principales operaciones tanto sintácticas como morfológicas que aparecen involucradas en la elaboración de construcciones causativas en el inglés criollo de Costa Rica.

\section{Construcciones causativas analíticas}

Estas construcciones implican la presencia de un verbo causativo independiente, que va precedido, por lo general, del agente de la causa y seguido del predicado del efecto, el cual constituye una cláusula independiente.

En el inglés criollo de Costa Rica, el verbo causativo más común y prototípico es MEK hacer. Dos ejemplos del uso de este verbo en construcciones causativas analíticas son los siguientes:

(1) Dayána mek im pikní laaf.

(Diana hacer ${ }^{2}$ su niño reír)

Diana hizo reír a su hijo.

(2) Man mek úman sófa.

(Hombre hacer mujer sufrir)

Los hombres hacen sufrir a las mujeres.

Como se ve en los ejemplos anteriores, el agente de la causa aparece categorizado como el sujeto del verbo causativo en la cláusula principal (Dayána + mek..., Man + mek...). El predicado del efecto aparece en la cláusula subordinada. En ella, el orden de los constituyentes es variable. En primer lugar, si se trata de una construcción intransitiva, entonces el orden de los constituyentes es el usual propio de esta lengua para las cláusulas independientes, es decir, primero el sujeto, que contiene al predicador del efecto, seguido del verbo, como se observa en las oraciones anteriores (im pikní + laaf, úman + sófa).

Cuando el predicador del efecto está representado por pronombres personales, estos pueden aparecer tanto en su forma de sujeto como en su forma de objeto.

(3) Im mek a $\left[=1^{\circ}\right.$ p. s. sujeto] tek bush.

(Él hacer yo tomar monte)

Él hizo que yo huyera. 
(4) Yu mek mi $\left[=1^{\circ}\right.$ p. s. sujeto u objeto $]$ laaf.

(Usted hacer yo reír)

Usted me hace reír.

(5) A mek shi $\left[=3^{\circ}\right.$ p. s. sujeto $]$ liiv tu nek.

(Yo hacer ella salir a cuello)

Hice que se fuera al infierno.

(6) A mek ar $\left[=3^{\circ}\right.$ p. s. objeto $]$ liiv tu nek.

(Yo hacer ella salir a cuello)

La mandé al infierno.

En primer término, una causa de esta alternancia puede atribuirse probablemente a la variación de formas propia del continuum post-criollo, típico de las lenguas criollas inglesas del Caribe. En las variedades más basilectales de criollo inglés de Costa Rica, la tendencia más usual es utilizar un mismo pronombre personal para las funciones de sujeto y objeto ${ }^{3}$ (el cual incluso es igual al pronombre posesivo).

Por otro lado, sin embargo, la diferencia en el uso del caso nominativo frente al acusativo para representar al predicador del efecto, utilizado en las variedades mesolectales, puede estar relacionado con el grado de control que ejerce el predicador del efecto sobre el evento causado. Si existe un alto grado de control por parte del predicador del efecto, entonces se utiliza el nominativo, que es un caso asociado prototípicamente con los agentes. Por el contrario, si el predicador del efecto tiene un control limitado sobre el evento causado, se usa el caso acusativo, que está relacionado más normalmente con pacientes. Es muy probable que esta diferenciación en el caso utilizado para representar al predicador del efecto se deba a una influencia del inglés estándar, como lengua de superestrato, en la que ocurre exactamente esta situación (cf. Payne 1997).

Con verbos transitivos, también existe la posibilidad de que el orden de los constituyentes sea el propio de esta lengua para las cláusulas independientes (Sujeto + verbo + Objeto).

(7) A mek mi pikní dem kliin dem ruum.

(Yo hacer mi niño PLU limpiar su cuarto)

Yo hago que mis hijos limpien su cuarto.

(8) A mek mi pikní waak di daag.

(Yo hacer mi niño pasear el perro)

Yo hice que mi hijo paseara al perro.

En las dos oraciones anteriores, el predicador del efecto en la cláusula subordinada se categoriza como sujeto (mi pikní) y el paciente lo hace como objeto directo (dem ruum, di daag). Hay que hacer notar, eso sí, que en estas oraciones la cláusula principal y la cláusula subordinada están unidas por yuxtaposición, sin la presencia de ninguna partícula subordinante o completizador.

En este tipo de construcciones, sin embargo, es posible también que aparezca un completizador delante del verbo de la cláusula subordinada. Este completizador es FI en las variedades más basilectales y TU, en las mesolectales. 
(9) Im mek shi fi stie uom.

(Él hacer ella COM quedarse casa)

La hizo quedarse en casa.

(10) Im mek ar tu elp im.

(Él hacer ella COM ayudar él)

Él la hizo ayudarlo.

(11) A mek yu fi óngri, fi mek yu fiil.

(Yo hacer vos COM tener-hambre para hacer vos sentir)

Te hice pasar hambre para que sintieras.

En las oraciones anteriores, es muy probable que el predicador del efecto funcione como objeto directo dentro de la cláusula principal. Esta operación de promoción del agente/sujeto de la cláusula subordinada a objeto de la cláusula principal parece evidente en (10), en la que la forma del pronombre AR indica que se trata de un objeto directo. Sin embargo, esta promoción no resulta tan evidente en (9), puesto que el pronombre SHI, funciona más prototípicamente como sujeto. Sin embargo, de nuevo hay que recordar que, en los basilectos, la tendencia preferida es utilizar el mismo pronombre para las funciones de sujeto y de objeto (cf. nota 3).

Es interesante señalar que el verbo causativo MEK se comporta en este respecto igual que otros de los verbos que introducen cláusulas subordinadas completivas, tales como AAKS pedir, TEL decir, WAAN querer, BEG rogar, OBLIGÍET obligar y KOMÁAN mandar.

(12) Shi waan mi fi go.

(Ella querer yo COM ir)

Ella quiere que yo me vaya.

(13) A obligíet mi pikní fi stódi.

(Yo obligar mi niño COM estudiar)

Obligué a mi hijo a que estudiara.

Por otro lado, con el verbo MEK, también es común unir la cláusula subordinada a la principal por medio de las conjunciones completizadoras DAT y SE, tanto en el caso de cláusulas subordinadas transitivas como intransitivas.

(14) A trai mek dat mi pikní wash di pliet.

(Yo tratar hacer COM mi niño lavar el plato)

Traté de hacer que mis hijos lavaran los platos.

(15) Ar mumá mek se shi kom rait ya.

(Su mamá hacer COM ella venir mismo aquí)

Su mamá hizo que viniera aquí mismo.

En este respecto, el verbo causativo MEK se comporta igual que los otros verbos que introducen cláusulas completivas antes mencionados. 
(16) Im tel ar dat shi mos go.

(Él decir a-ella COM ella MOD-DEB ir)

Él le dijo que (ella) debía irse.

(17) Shi waan dat mi go.

(Ella querer COM yo ir)

Ella quiere que yo me vaya

En este tipo de construcciones completivas introducidas por los completizadores DAT y SE, es posible realizar una operación sintáctica de topicalización de argumentos distintos del sujeto $^{4}$. Esta consiste en colocar un argumento (o un adyacente) inmediatamente después del completizador y delante del sujeto de la cláusula subordinada. Se trata, pues, de una operación de anteposición (fronting) ${ }^{5}$ de un argumento al inicio de la cláusula, de un cambio en el orden normal de los constituyentes de la frase (cf. Payne 1997: 271 y ss.).

(18) A mek dat di pliet mi pikní dem wash i.

(Yo hacer COM el plato mi niño PLU lavar ello)

Yo hago que los platos los laven mis hijos.

(19) A mek dat di fluor mi pikní kliin.

(Yo hacer COM el piso mi niño limpiar ello)

Hice que el piso lo limpiara mi hija.

En las oraciones anteriores, el objeto directo de las cláusulas subordinadas (di pliet, di fluor) aparece antes del sujeto (mi pikní dem, mi pikní). Es interesante señalar que el objeto directo puede repetirse nuevamente dentro de la cláusula subordinada en el lugar que le corresponde después del verbo, representado entonces por un pronombre personal, como en (18), o puede no hacerlo, como en (19).

No solamente puede topicalizarse el argumento de objeto directo, sino que también lo puede hacer el argumento de objeto indirecto, como en (20), (21) y (22).

(20) A mek dat Mierí im gi di móni.

(Yo hacer COM María él dar el dinero)

Hice que a María le diera la plata.

(21) A mek dat fi dem mumá dem bai a prézant.

(Yo hacer COM para su (= de ellos) mamá ellos comprar un regalo)

Hice que a su mamá le compraran un regalo.

(22) A mek dat dem mumá dem bai a prézant fi.

(Yo hacer COM su (= de ellos) mamá ellos comprar un regalo para)

Hice que a su mamá le compraran un regalo.

La comparación de (21) y (22) pone en evidencia que, cuando el objeto indirecto está representado por una frase preposicional, la preposición puede ser trasladada o no junto con la frase nominal que está topicalizándose. 
Argumentos o adyacentes que corresponden a adverbios o frases preposicionales de valor circunstancial también pueden topicalizarse, siendo promocionados a la primera posición de la cláusula subordinada, como en (23), (24) y (25).

(23) A mek dat yéside im kom fi taak tu im.

(Yo hacer COM ayer él venir para hablar a él)

Hice que ayer viniera para hablar con él.

(24) A mek dat wid a bruum mi pikní swiip di mes.

(Yo hacer COM con una escoba mi niño limpiar el reguero)

Hice que con una escoba mi hijo limpiara el reguero.

(25) A mek dat tu Limón im gaan.

(Yo hacer COM a Limón él irse)

Hice que a Limón se fuera.

Por otro lado, es importante señalar que esta operación de anteposición es distinta de otra regla de extraposición a la izquierda (left-dislocation) (cf. Payne 1997: 273) que, en esta lengua, tiene una función focalizadora o de contraste ${ }^{6}$. La operación de extraposición también permite trasladar cualquier argumento al inicio de la cláusula, pero este debe estar precedido del marcador $\mathrm{A}^{7}$, como se nota en las cláusulas simples (26) y (27).

(26) A dis buk a don riid it aredi.

(FOC este libro yo COM leer ello ya)

Este libro [no otro] yo ya me lo leí.

(27) A wan jab mi niid, no yu móni.

(FOC un trabajo yo necesitar no tu dinero)

Un trabajo es lo que necesito, no tu plata.

Los ejemplos (28) y (29) demuestran que esta regla de focalización es distinta de la regla de topicalización mencionada antes.

(28) A mek dat di pliet a mi pikní dem wash i.

(Yo hacer COM el plato FOC mi niño PLU lavar ello)

Yo hago que los platos sean mis hijos [no yo] los que los laven.

(29) A mek dat di kluoz a mi pikní dem eng i.

(yo hacer que la ropa FOC mi niño PLU colgar ello)

Yo hago que la ropa sean mis hijos [no yo] los que la tiendan.

La oración (28) presenta la aplicación de las operaciones de anteposición y de extraposición a la izquierda, mientras que en (18) citada anteriormente solo se había aplicado la operación de anteposición. Finalmente, se puede agregar que resulta agramatical la cláusula en la que se pretenda focalizar un argumento que ya ha sido topicalizado. 
(30) * A mek dat a wid di pan im tek op di wáata.

(Yo hacer COM FOC con la palangana él recoger el agua)

Hice que fuera con la palangana [no con otra cosa] que él recogiera el agua.

Por otra parte, es interesante señalar la existencia de una construcción causativa analítica que carece de un agente explícito de la causa, pero que sí presenta el verbo causativo MEK y el predicado del efecto en una cláusula independiente.

(31) Mek wi go.

(Hacer nosotros ir)

¡Vámonos!

(32) Mek a tel yu somtíng.

(Hacer yo decir te algo)

Dejáme decirte algo.

(33) A táyad, mek a se.

(Yo estar-cansado hacer yo decir)

Estoy cansado, déjeme que le diga.

(34) No mek im krai

(Hacer él llorar)

No lo haga llorar.

(35) Mek shi shot op.

(Hacer ella callarse)

Hagan que se calle.

Este tipo de construcciones causativas expresan predicaciones de modalidad imperativa. En las oraciones (32), (33) y (34), aunque no aparezca explícitamente el agente de la causa, se puede suponer que este agente corresponde a la segunda persona singular (YU, vos/usted); mientras que en las oraciones (31) y (35), el agente concierne a la segunda persona plural (UNU, ustedes). De ser así, la ausencia explícita del agente de la causa se debe simplemente a la omisión del sujeto de un verbo en modo imperativo, que es lo usual en esta lengua.

$$
\begin{aligned}
& \text { kom ya! } \\
& \text { (venir aquí!) } \\
& \text { ¡Venga acá! }
\end{aligned}
$$

En esta lengua, existe también una construcción causativa analítica que expresa una causación indirecta. En esta construcción, el agente de la causa interviene de una forma más atenuada, actuando más bien como un instigador del evento que expresa el efecto. El verbo causativo más prototípico utilizado en esta construcción es GET lograr, conseguir. 
(37) Jan get Mierí go wid im.

(Juan lograr María ir con él)

Juan logró que María saliera con él.

(38) Im get Mierí riid di buk.

(Él lograr María leer el libro)

Él logró que María leyera el libro.

En las construcciones causativas indirectas también pueden utilizarse facultativamente los completizadores FI (TU) y SE (DAT) de la misma forma que se ha mencionado antes.

(39) Lorna get di bíebi fi sliip.

(Lorna lograr el bebé COM dormir)

Lorna logró que el bebé se durmiera.

(40) Shi get dat Ándru iit op di dína.

(Ella lograr COM Andrés comer todo la comida)

Ella logró que Andrés se comiera toda la comida.

Por otro lado, también, en este tipo de construcciones se puede aplicar la operación de anteposición descrita anteriormente para las causativas directas.

(41) Shi get dat ar Jan kaal fors.

(Ella lograr COM ella Juan llamar primero)

Ella logró que a ella la llamara Juan primero.

Por último, también ocurre que cuando se utilizan pronombres personales para representar el predicador del efecto en una cláusula subordinada intransitiva, estos puedan aparecer o bien en caso nominativo, como sujetos, o bien en caso acusativo, como objetos directos, tal como se señaló antes para las causativas directas con el verbo MEK.

(42) Im get shi go.

(Él lograr ella ir)

Él logró que ella se fuera.

(43) Im get ar go.

(Él lograr ella ir)

Él logró que ella se fuera.

\section{Construcciones causativas léxicas}

Desde el punto de vista tipológico, existen al menos tres clases de causativos léxicos (Payne 1997:177). En todos los casos, la noción de la causa está expresada totalmente por el significado léxico del verbo. Los diferentes tipos están relacionados con la forma que toma el 
verbo causativo respecto de un verbo no causativo que expresa un contenido semántico idéntico a aquel. Los tres tipos de verbos se caracterizan de la siguiente manera: (1) el verbo causativo presenta una forma idéntica al verbo no causativo, (2) el verbo causativo sufre algún cambio idiosincrático de forma respecto del verbo no causativo y (3) el verbo causativo es totalmente diferente del verbo no causativo.

En el primer tipo, se podría decir que se ha aplicado una operación cero ${ }^{8}$ para formar un verbo causativo a partir de un verbo no causativo. La operación cero es el caso de formación de causativos más común en esta lengua, lo cual se debe seguramente a la ya mencionada carencia de morfología derivativa. Esta operación permite convertir verbos de proceso, de estado e, incluso, de acción en verbos de acción-proceso.

Los verbos de proceso, estado y acción, por lo general, implican solamente la presencia de un paciente o un agente, según el caso, mientras que los verbos de acción-proceso añaden un participante más (el agente de la causa) a la escena que la predicación expresa.

Con verbos de proceso:

(44 a) Di plant a gruo.

CONSTRUCCIÓN NO CAUSATIVA

(La planta PRO crecer)

Las plantas están creciendo.

(44 b) Rien a gruo di plant.

CONSTRUCCIÓN CAUSATIVA

(Lluvia PRO crecer la planta)

La lluvia está haciendo crecer las plantas 9 .

(45 a) Úman sófa alát.

CONSTRUCCIÓN NO CAUSATIVA

(mujer sufrir mucho)

Las mujeres sufren mucho

(45 b) Man sófa úman tu det.

CONSTRUCCIÓN CAUSATIVA

(Hombre sufrir mujer a muerte)

Los hombres hacen sufrir mucho a las mujeres.

Con verbos de estado:

(46 a) Man fuul.

CONSTRUCCIÓN NO CAUSATIVA

(Hombre ser-tonto)

Los hombres son tontos.

(46 b) Úman fuul man

CONSTRUCCIÓN CAUSATIVA

(Mujer idiotizar hombre)

Las mujeres idiotizan a los hombres.

(47 a) A ríili táyad.

CONSTRUCCIÓN NO CAUSATIVA

(Yo realmente estar-cansado)

Estoy realmente cansado. 
(47 b) Líetli mi sísta a táyad mi. CONSTRUCCión CAUSATIVA

(Últimamente mi hermana PRO cansar me)

Mi hermana me está cansando últimamente.

Con verbos de acción:

(48 a) Shi taak tumóch.

CONSTRUCCIÓN NO CAUSATIVA

(Ella hablar demasiado)

Ella habla demasiado.

(48 b) Im a taak mi kózin.

CONSTRUCCIÓN CAUSATIVA

(Él PRO hablar mi prima)

Él está cuenteando a mi prima.

(49 a) Somtáimz yu afi ron.

CONSTRUCCIÓN NO CAUSATIVA

(A-veces usted MOD-DEB correr)

A veces uno tiene que correr.

(49 b) Im ron im daag outsaid.

CONSTRUCCIÓN CAUSATIVA

(Él correr su perro afuera)

Echó al perro puerta afuera.

En todas las oraciones causativas anteriores, aparece un participante más que en las oraciones no causativas. La forma del verbo no causativo es, sin embargo, idéntica a la que presenta el verbo causativo.

Es interesante mencionar que la interpretación del significado del verbo depende precisamente del contexto pragmático en el que se encuentre la predicación. En este sentido, de manera general, la interpretación semántica de una cláusula depende absolutamente del contexto. Tal es el caso de la cláusula (50) (tomada de Portilla 1998: 174), cuya interpretación intransitiva, reflexiva, media o pasiva no puede hacerse con certeza fuera de contexto, a pesar de que en todos los casos el sujeto corresponda a un paciente bastante prototípico y el verbo sea de acción-proceso.

(50) A biit. (Yo golpear)

Yo gané (en un juego)

Me pegué a mí mismo (de rabia)

INTERPRETACIÓN INTRANSITIVA

Me golpeé (involuntariamente)

INTERPRETACIÓN REFLEXIVA

Fui golpeado por alguien

INTERPRETACIÓN MEDIA

INTERPRETACIÓN PASIVA

En el segundo tipo, el verbo causativo sufre algún cambio de forma idiosincrático respecto del verbo no causativo. Se trata, pues, de una operación morfológica de reemplazamiento vocálico o consonántico. Se puede afirmar que este tipo de formación de causativos no ocurre prácticamente en esta lengua. 
Los lexemas que en inglés estándar presentan un proceso de reemplazamiento vocálico para la formación de causativos, no se corresponden de la misma manera a sus respectivos cognados en este criollo. En inglés estándar, existe una serie reducida de verbos (cf. Givón 1990: 556 y Payne 1997:177) cuya alternancia vocálica permite distinguir un no causativo (intransitivo) de un causativo (transitivo). Así, por ejemplo, los verbos no causativos RISE [raiz] levantarse, ponerse de pie, FALL [fol] caer, caerse, SIT [sit] sentarse, estar sentado y LIE [laI] acostarse, echarse se correlacionan, por medio de una alternancia vocálica, respectivamente con los verbos causativos RAISE [reIz] levantar, FELL [fel] talar, derribar, SET [set] poner, colocar y LAY [leI] poner, colocar. En inglés criollo, los verbos REIZ, FAAL, SIT y LIE (que corresponden a los cognados del estándar RAISE, FALL, SIT y LAY ${ }^{10}$ ) poseen un significado tanto causativo como uno no causativo.

El verbo REIZ abarca el significado del causativo RAISE y del no causativo RISE, tal como se nota en los siguientes ejemplos.

(51 a) Di man riez an gaan.

CONSTRUCCIÓN NO CAUSATIVA

(El hombre levantarse y irse)

El hombre se levantó y se fue.

(51 b) Riez di bíebi fram kuul kyáapit. CONSTRUCCión CAUSATIVA (Levantar el bebé de fría alfombra)

Levante al chiquito del linolio.

Por su parte, el verbo FAAL comprende el significado del causativo FELL y del no causativo FALL.

(52 a) Di trii faal fram di stráikin. CONSTRUCCión no CAUSATIVA

(El árbol caer de la rayería)

El árbol se cayó por la rayería.

(52 b) Di munísipi faal aal di trii dem out a di paak. Construcción CAUSATIVA (La municipalidad caer el árbol PLU fuera de el parque)

La municipalidad derribó todos los árboles del parque.

En inglés criollo, el verbo siT presenta un sentido no causativo en cláusulas intransitivas, como en inglés estándar; pero también puede usarse con un significado causativo en cláusulas transitivas, a diferencia del estándar.

(53 a) Jan a sit dong out de.

CONSTRUCCIÓN NO CAUSATIVA

(Juan PRO estar-sentado abajo fuera ahí)

Juan está sentado ahí afuera.

(53 b) Sit yu aas pan di dyam chier an no muuv it fram dier.

CONSTRUCCIÓN CAUSATIVA

(Sentar tu trasero en la maldita silla y no mover ello de ahí)

Acomode su trasero en la bendita silla y no lo mueva de ahí. 
También, el verbo LIE en criollo abarca tanto el sentido no causativo de LIE como del causativo LAY, del inglés estándar.

(54 a) Di daag lie pan di súofa.

CONSTRUCCIÓN NO CAUSATIVA

(El perro estar-echado en el sofá)

El perro está echado en el sofá.

(54 b) Jan lie di daag fuud said a im.

CONSTRUCCIÓN CAUSATIVA

(Juan poner la perro comida junto de él)

Juan puso la comida del perro a la par de él (del perro)

Las formas correspondientes a los verbos RICE, FELL y LIE no se utilizan normalmente en esta variedad criolla. Su eventual aparición puede atribuirse a la influencia de superestrato que el inglés estándar ejerce sobre el criollo ${ }^{11}$. El verbo SET es usado frecuentemente en criollo; pero a diferencia del estándar, este puede aparecer también en construcciones intransitivas ${ }^{12}$.

En el tercer tipo, el verbo causativo es totalmente diferente del verbo no causativo. En este caso, se podría hablar de la aplicación de una operación morfológica de reemplazamiento total o de supleción. Este tipo de formación de causativos léxicos permite que verbos de proceso, de estado y de acción se conviertan en verbos de acción-proceso. Algunos ejemplos de este tipo de correlación entre parejas de verbos no causativos y causativos son los siguientes:

WAIT palidecer (no causativo, de proceso) /

PIEL hacer palidecer (causativo, de acción-proceso)

(55 a) Ar fies wait wen shi si im.

Construcción NO CAUSATIVA

(Su cara palidecer cuando ella ver él)

Se le puso la cara pálida cuando lo vio

(55 b) Di nyuuz piel Mierí.

CONSTRUCCIÓN CAUSATIVA

(La noticia palidecer María)

La noticia hizo palidecer a María.

DED estar-muerto (no causativo, de estado) /

KILL matar (causativo, de acción-proceso)

(56 a) Di spáida ded.

CONSTRUCCión NO CAUSATIVA

(Araña estar-muerto)

La araña está muerta.

(56 b) A no laik fi kil spáida.

CONSTRUCCIÓN CAUSATIVA

(Yo no gustar COM matar araña)

No me gusta matar arañas.

SI ver (no causativo, de acción) /

SHUO mostrar (causativo, de acción-proceso) 
(57 a) Díevid si di pícho wid mi.

CONSTRUCCIÓN NO CAUSATIVA

(David ver la película con mi)

David vio la película conmigo.

(57 b) Píita shuo im kyar tu Diego.

CONSTRUCCIÓN CAUSATIVA

(Pedro mostrar su carro a Diego)

Pedro le enseñó el carro a Diego.

En algunos casos, el verbo supletivo con sentido causativo parece surgir por una evidente influencia del inglés estándar, que, como lengua de superestrato, provee vocabulario nuevo a este criollo. Así, por ejemplo, los verbos causativos FIID alimentar y тIICH enseñar, que forman pareja con los verbos no causativos IIT comer y LORN aprender, respectivamente, son sustituidos por las formas verbales GI FUUD dar comida, GI FI IIT dar de comer y LORN aprender/ enseñar ${ }^{13}$, en las variedades criollas más basilectales.

(58 a) Yu fiid di chíkin? ${ }^{14}$

(¿Vos alimentar la gallina?)

¿Alimentaste las gallinas?

(58 b) A gi di foul fi iit.

(Yo dar la gallina COM comer)

Les di de comer a las gallinas.

(59 a) A gwain tiich yu Spánish.

(Yo IRR enseñar te español)

Te voy a enseñar español.

(59 b) A gwain lorn yu a lésin.

(Yo IRR enseñar te una lección)

Te voy a dar una lección.

\section{Conclusiones}

En el inglés criollo de Costa Rica, las predicaciones causativas pueden expresarse, fundamentalmente, de tres formas diferentes.

Una construcción analítica en la que utiliza el verbo MEK manifiesta una intervención más directa del agente de la causa sobre el evento que se predica en el efecto.

(60) Im mumá mek im stódi.

(Su mamá hacer él estudiar)

Su mamá lo hizo estudiar.

Una construcción analítica en la que aparece el verbo GET indica que la influencia del agente de causa sobre el evento expresado en la predicación del efecto es indirecta. 
Esto implica que el predicador del efecto, en la cláusula subordinada, tiene mayor autonomía respecto de la influencia del agente de la causa.

(61) Ántil, a get yu stódi.

(Finalmente yo lograr vos estudiar)

Finalmente, logré que estudiaras.

Una construcción causativa léxica frente a una causativa analítica expresa una influencia físicamente más directa del agente de la causa sobre el predicador del efecto. Esto aparece evidente en la comparación de (62) y (63).

(62) Dat pikní kil im pet.

(Ese niño matar su mascota)

Ese niño mató a su mascota.

(63) Dat pikní mek im pet ded.

(Ese niño hacer su mascota morir)

Ese niño hizo que su mascota se muriera.

La oración (62) muestra una intervención más directa del agente de la causa, PIKNí, el cual pareciera manifestar voluntad y un mayor control de la situación. Por otra parte, en la oración (63), la influencia del agente de la causa parece ser físicamente indirecta e incluso dar a entender un menor control de la situación y falta de conciencia y voluntad por parte del agente de la causa sobre el predicador del efecto.

Las oraciones (64) y (65) reflejan una situación similar.

(64) Jan mek Mierí si di fúoto.

(Juan hacer María ver la foto)

Juan hizo que María viera las fotos.

(65) Jan shuo Mierí di fúoto.

(Juan mostrar María la foto)

Juan le mostró a María las fotos.

El hecho de que en (65) el argumento MiERí se halle categorizado como un objeto indirecto (semánticamente, el participante se categoriza como un beneficiario de la acción) pone en evidencia que el control que puede ejercer el agente de la causa sobre un beneficiario es mayor. Por el contrario, en (64), se categoriza a MIERí como un sujeto, el cual prototípicamente corresponde a un agente, con voluntad y cierta autonomía. Esto significa, entonces, que la influencia del agente de causa sobre el predicador del efecto sería menor.

Por supuesto, también es posible que objetos inanimados puedan aparecer como agentes de la causa en construcciones analíticas, lo mismo que en construcciones causativas léxicas. La diferencia semántica entre las causativas analítica y léxica expresadas en (66) radicaría precisamente en el énfasis de la menor (66 a) o mayor (66 b) influencia que se le atribuye al agente de la causa sobre el predicador del efecto. 
(66 a) Télivishan mek pikní sliip.

(Televisión hacer niño dormir)

La televisión hace dormir a los niños.

(66 b) Télivishan sliip pikní.

(Televisión dormir niño)

La televisión duerme a los niños.

Por otro lado, es muy importante señalar que uno de los aspectos más notables de esta lengua en relación con las construcciones causativas léxicas es el hecho de que verbos prototípicamente no causativos puedan funcionar como causativos sin ningún cambio de forma, a pesar de que los cognados correspondientes en inglés estándar no puedan de ninguna manera funcionar de la misma forma. Igualmente, verbos prototípicamente transitivos pueden aparecer en construcciones intransitivas.

Otro par de ejemplos que se pueden agregar a los ya mencionados desde (37) hasta (47) son los siguientes:

(67 a) Mierí did a laaf bikáaz a Jan juok. COnSTRUCCión no CAUSATIVA (María reír a-causa de Juan chiste)

María se estaba riendo por los chistes de Juan.

(67 b) Jan laaf Mierí tu det.

CONSTRUCCIÓN CAUSATIVA

(Juan reír María a muerte)

Juan mató de risa a María.

(68 a) Évribadi beks wid im.

CONSTRUCCIÓN NO CAUSATIVA

(Todos estar-enojado con él)

Todos estaban enojados con él.

(68 b) Im laik beks im wórka front a píipl. CONSTRUCCIÓn CAUSATIVA

(Él gustar enojar su esposa frente a gente)

A él le gusta enojar a su esposa delante de las personas.

Como se ha dicho en otra ocasión (cf. Portilla 1998), es probable que esta situación sea producto de una tendencia a la simplificación propia de las lenguas criollas, en el sentido de una utilización óptima de los recursos léxicos y morfosintácticos.

\section{Abreviaturas}

$\begin{array}{ll}\text { COM } & \text { Completizador } \\ \text { FOC } & \text { Marcador de foco o contraste } \\ \text { IRR } & \text { Modo irrealis } \\ \text { MOD-DEB } & \text { Modal deóntico de deber } \\ \text { OD } & \text { Objeto directo } \\ \text { PLU } & \text { Plural } \\ \text { PRO } & \text { Aspecto progresivo }\end{array}$




\section{Notas}

1. En esta fórmula, ' $\mathrm{x}$ ' representa una variable, es decir, un término que no tiene una referencia específica. Esta variable puede, por supuesto, ser sustituida por una constante, es decir, un término de referencia específica, como un nombre propio u otras frases nominales. ' $\mathrm{P}$ ' representa una proposición, es decir, un predicado que se aplica a una constante.

2. El verbo en su forma simple puede tener una interpretación semántica de pasado o presente según el contexto. Para una descripción más completa de estos usos, cf. Portilla 1996.

3. Los pronombres personales utilizados en los basilectos para ambas funciones son los siguientes:

$\begin{array}{cll} & \text { singular } & \text { plural } \\ 1^{\circ} & \mathrm{mi} & \text { wi } \\ 2^{\circ} & \text { yu } & \text { unu } \\ 3^{\circ} \mathrm{m} . & \mathrm{im} & \text { dem } \\ \mathrm{f} . & \text { shi } & \\ \mathrm{n} . & \mathrm{i} & \end{array}$

Los pronombres personales utilizados en los mesolectos se distinguen, además, por caso, diferenciando entre nominativo y acusativo, aunque no en todas las personas gramaticales:

\begin{tabular}{cllll}
\multicolumn{3}{c}{ singular } & \multicolumn{3}{c}{ plural } \\
& nom. & ac. & nom. & ac. \\
$1^{\circ}$ & $a$ & $m i$ & wi & as \\
$2^{\circ}$ & yu & yu & unu & unu \\
$3^{\circ}$ m. & $i i$ & im & dem & dem \\
f. & shi & ar & & \\
n. & it & it & &
\end{tabular}

4. Para nuestra exposición, son pertinentes dos de los sentidos que Payne (1997: 270) ofrece como definiciones del término tópico: 1. The topic is a dislocate clause constituent. Sometimes such elements are termed "topicalization", and the pragmatically marked structures that encode them "topicalization" 2. The topic is a clause-level notion that can be paraphrased "what the clause is about."

5. Otro término utilizado para describir esta operación formal es movimiento-Y (Y-movement) (cf. Givón 1990: 705).

6. En el nivel de la cláusula, la focalización contrastiva permite al hablante afirmar la identidad correcta de una entidad que posee un rol particular en un determinado evento, cuando percibe que el oyente podría atribuir a otra entidad el papel o rol que a aquella le correspondía.

7. El marcador $a$ es, por cierto, idéntico a la cópula ecuativa, en esta lengua.

8. $\quad$ Esta terminología está tomada de Mattews 1980.

9. Cf. esta construcción causativa léxica con la correspondiente construcción causativa analítica:

Rien a mek di plant gruo.

(Lluvia PRO hacer la planta crecer)

La lluvia está haciendo crecer las plantas.

10. El verbo LIE en inglés criollo proviene de la forma LAY, que es el pretérito del verbo intransitivo LIE, y no del verbo transitivo homófono LAY.

11. En inglés criollo, el verbo RAIz levantarse/ levantar, aunque ciertamente no presenta un sentido causativo, connota la utilización de un estilo solemne y puede aparecer en cláusulas transitivas. 
A gwain raiz mai vaiz agéns évil

(Yo IRR levantar mi voz contra mal)

Voy a levantar la voz contra el mal.

Además, puede ser intercambiado por el verbo RIEZ, incluso en construcciones típicamente intransitivas.

Di son raiz at siks.

(El sol levantarse a seis)

El sol sale a las seis.

Di son riez at siks.

(El sol levantar a seis)

El sol sale a las seis.

El verbo FEL siempre es interpretado como la variante acrolectal con significado de pasado del verbo FAAL. El verbo LAI también se interpreta como una variante, más bien acrolectal, del verbo LIE. En este caso, el verbo LAI puede aparecer tanto en cláusulas intransitivas (al igual que el verbo LIE en estándar) como transitivas (a diferencia del estándar):

Jan lai dong insáid.

CLÁUSULA INTRANSITIVA

(Juan estar-acostado adentro)

Juan está acostado adentro.

Im no lai dong it de an gaan?

CláUSULA TRANSITIVA

(Él no poner abajo ello ahí y irse?)

¿No lo puso ahí y se fue?

12. Un ejemplo de un uso intransitivo de este verbo es el siguiente:

Di buk dem set niit niit pan di ráitin tíebl.

(El libro PLU estar-colocado ordenado ordenado en la escribir mesa)

Los libros están acomodados impecablemente en el escritorio.

13. El sentido de lorn (< learn) como enseñar, aunque ahora tiene un carácter no estándar, es común en inglés desde el siglo XII (cf. el OED).

14. En inglés criollo, la palabra más común utilizada para referirse a las gallinas es FOUL (< FOWL, a domestic cock or hen, actualmente dialectal, OED), mientras que la palabra cHÍKIN es más bien un término acrolectal.

\section{Bibliografía}

Givón, T. 1984. Syntax. Amsterdam: Benjamins.

Mattews, P. H. 1979. Morphology. Cambridge: Cambridge University Press.

Payne, T. 1997. Describing morphosyntax. Cambridge: Cambridge University Press.

Portilla, M. 1996. "Tiempo, aspecto y modo en el criollo inglés de Costa Rica”. Revista de Filología y Lingüística de la Universidad de Costa Rica. 23 (1): 224-32.

1998. "Operaciones de reducción de valencia en el criollo inglés de Costa Rica". Revista de Filología y Lingüística de la Universidad de Costa Rica. 24 (2): 167-76. 